\title{
Hypovolemic shock
}

\begin{abstract}
Hypovolemic shock is a complex condition very prevalent in our time. It is associated with high mortality and a prompt intervention could save lifes. Mortality is related to severity of trauma and bleed lost, management quality and time from trauma to therapy. In this review from the last 10 years of literature some aspects of pathophysiology, monitoring and treatment will be presented.
\end{abstract}

Keywords: hemorrhagic shock, hemodynamic monitoring, pathophysiology
Volume 2 Issue 3 - 2017

\author{
Claudio Piras \\ Department of Surgical Clinic, Federal University of Espírito \\ Santo, Brazil
}

Correspondence: Claudio Piras, Department of surgical clinic, Federal University of Espírito Santo, Brazil,Tel +552798 I I I 3955, Email cpiras@uol.com.br

Received: November 09, 2017 | Published: December 08, 2017

\section{Introduction}

Shock is one of the most complex conditions that can be found in critical patients and is associated with a high mortality distributed in early (hemorrhagic shock), postinjury (trauma brain injury) and late (organ failure). ${ }^{1}$ The term "shock" encompasses a wide range of pathological processes that may require diametrically opposed methods of treatment. It is defined as a life-threatening circulatory failure where the oxygen delivery is not enough to tissue demand. ${ }^{2}$ The underlying cause may be easily identified as in the traumatic shock resulting from massive hidden blood loss. Shock is a microcirculatory derangement associated with hypoperfusion and celular death. ${ }^{3}$ Shock respond differently to different therapies because is associates to many physiological alterations caused by increased vascular permeability, interstitial edema and intravascular volume depletion,with classic signs including hypotension, decreased urine output, and multiple organ insufficiency. ${ }^{4}$

Hypovolemic shock is an important cause of morbidity and mortality. Rapid diagnosis associated with immediate appropriate therapy is essential for survival. Prompt hemodynamic stabilization is the basis of initial treatment. ${ }^{5}$ Here some conceptual, physiological, and pathophysiological aspects of hypovolemic shock will be presented including monitoring and basics concepts of treatment.

\section{Methods}

Data was obtained from Pubmed using the key words hemorrhagic shock, hypovolemic shock, monitoring, pathophysiology, trauma and hypoperfusion. Data from the last 10 years was checked for suitability.

\section{Definitions}

Shock is a state of inadequate tissue perfusion and oxygenation characterized by an unbalance between oxygen supply (DO2) and demand (VO2), which will lead to the injury of body tissues. ${ }^{3,5,6}$ Arterial hypotension may be present, but it is not essential in the diagnosis of shock. Special attention should be on signs of tissue hypoperfusion such as changes in mental state; cardiac changes, such as tachycardia; and kidney alterations, such as oliguria in patients without renal disease. ${ }^{4,7}$ Hypovolemic shock is characterized by inadequate cardiac output due to insufficient circulating volume, as seen in bleeding, pancreatitis, and large burns., ${ }^{4,-10}$

\section{Pathophysiology}

Hypovolemia is the reduction of circulating blood volume. Hypovolemia decreases the filling pressure and the Heart Rate to values below that necessary to maintain tissue perfusion, a situation known as shock. The decrease in blood pressure (BP) leads to a neuroendocrine response via the autonomic nervous system, where adrenaline and noradrenaline released in the circulation increase cardiac contractility, heart rate (HR) and promote vasoconstriction, triggered mainly by baroreceptors and low pressure vascular stretching receptors. It also stimulates the renin-angiotensin system, releasing angiotensin II, a potent vasoconstrictor, and stimulates the secretion of aldosterone, which promotes the conservation of water and salt. Increased adrenergic activity also favors the flow of some organs like brain, myocardium, and adrenals. In the acute phase of the disease, there is also an increase in cortisol production due to the secretion of corticotrophin releasing hormone (CRH), ACTH (adrenocorticotrophic hormone) and reduction of cortisol by negative feedback. . $, 4,7,11-14$ When BP falls to a sufficiently low level, there is also a decrease in coronary blood flow, which decreases the contractile capacity of the heart muscle and thus further decreases the DC, making the shock increasingly severe. Blood stagnation in the microvessels and tissue metabolism continues to increase. A large amount of acid (carbonic and lactic acid) continues to be produced and released into the local blood vessels, increasing the acidity of blood with other products of ischemic tissue degradation, lead to blood clumping, clot formation and consequent obstruction of microvascular bed. After a few hours of hypoxia, there is an increase in capillary permeability and a large amount of fluid begins to flow to the tissues and edema formation. ${ }^{7,11-14}$

Shock affects many systems with various signs of cellular degeneration like: Less active transport of sodium and potassium through the cellular membrane; Depression of the mitochondrial activity; Lysosomes dysfunction, with release of hydrolases and additional cell injury; and Depression of cellular metabolism of nutrients, such as glucose, as well as the activity of some hormones, such as insulin. ${ }^{7}$

\section{Hemodynamic aspects}

The quantitative determination of hemodynamic function is essential in the proper handling of the patient in hypovolemic 
shock. Since preload reduction (volemia) is the underlying cause of hypovolemic shock, volume replacement based on clinical parameters is essential in treatment. ${ }^{11}$ However, clinical observation alone in these patients is often subjective and has a low correlation with the hemodynamic parameters measured. Data from the literature show that the intensivist is unable to accurately predict variables such as cardiac output (DC), occluded pulmonary artery pressure (PAPO) and peripheral vascular resistance (SVR) in more than $50 \%$ of events, despite having access to central venous pressure (PVC) and radiological examinations. ${ }^{15}$ These data reinforce the need to accurately determine preload in these patients. Thus, invasive hemodynamic monitoring is a fundamental part of handling the patient in shock. . $^{11,12,15-23}$

The main hemodynamic variables in hypovolemic shock are: arterial pressure (BP), heart rate (HR), central venous pressure (CVP), occluded pulmonary artery pressure (PAPO), cardiac output (DC), cardiac index (VVF), right ventricular end-diastolic volume (VDFG), variation of systolic pressure (VPS), variation of pulse pressure (PPV), systolic volume variation (VVS) and plethysmographic waves variation (VOP)..$^{15-25}$ Variables are divided into static or dynamic discussed below.

\section{Static variables}

Static variables can be divided into pressure and volume variables. Pressure variables include PVC and PAPO. ${ }^{26}$ Volume variables included left ventricular end-diastolic volume (LVEDV) and overall final diastolic volume (LVSD). ${ }^{16,17,20}$ The VDFVE is the volume of blood within the left ventricle at the end of the diastole. It is measured indirectly through transesophageal echocardiography. Its normal value in healthy non-athlete adults is 152 to $176 \mathrm{ml}^{7}$ and is decreased in hypovolemic shock due to decreased preload. VDFVE has not been able to effectively predict fluid responsiveness. ${ }^{16,26}$ Another volumetric static variable is VDFG, which is measured by transpulmonary thermodilution, is also reduced in hypovolemic shock. This method has been considered a pre-load indicator and a possible predictor of volume responsiveness. Some studies have shown good sensitivity and specificity of this method, while others suggest a lack of correlation between the measured values and the change in cardiac output. ${ }^{16,26}$

\section{Dynamic variables}

Dynamic variables are based on the intermittent decrease in venous return due to the increase in intrathoracic pressure produced by positive pressure mechanical ventilation cycles. With decreased venous return, the left ventricular preload also decreases. The analysis of this effect on systolic volume or relative variables such as pulse pressure or systolic pressure has been the main concept of functional hemodynamic monitoring. ${ }^{17,20-23,27}$

The systolic pressure variation (VPS) evaluates the difference between the maximum systolic pressure and the minimum systolic pressure throughout a respiratory cycle. A difference greater than the normal value of 8 to $10 \mathrm{mmHg}$ is an accurate indicator of hypovolemia. ${ }^{17,20-23,27}$ Pulse Pressure Variation (PPV) and Systolic Volume Variation (VVS) are based on the measurement of systolic volume or pulse pressure, derived from the pulse contour. Values equal to or greater than $13 \%$ of pulse pressure variation showed a good response predictor (increase in cardiac output) to volume replacement. The variation in systolic volume under normal conditions is 5 to 10 $\mathrm{mmHg}$. Values greater than $10 \mathrm{mmHg}$ have been related to patients responsive to volume replacement. ${ }^{17,20-23,27}$
Variation of the plethysmographic waveform measures the amplitude variation of the plethysmographic waveform of pulse oximetry. The measure is considered positive for volume responsiveness when it reaches variation of $10 \%$ for automatic measures and $12 \%$ for manual measures. ${ }^{17,20-23,27}$

\section{Management}

The treatment of hemorrhagic shock, which is the most common cause of shock in trauma, comprises interruption of bleeding and volume replacement, whith blood and its derivatives or solutions, like crystalloids or colloids. The most used component in volume replacement is crystalloid solutions, in order to reestablish cardiac output and tissue perfusion. This practice, although well accepted, is not free of complications. Crystalloids and colloids have been associated with inflammation, hemodilution, edema, abdominal compartment syndrome, renal failure and coagulation disorder. ${ }^{14,28-31}$ Fluid replacement can be monitored by dynamic methods with advantages over static variables and vital signs. ${ }^{23}$

In cases of severe bleeding and massive blood replacement, some criteria observed in recent years should be taken into account: loss of blood viscosity, coagulation changes due to hemodilution and fibrinolysis. For volumetric replacement that prevents severe impairment of tissue perfusion, data retrieved from recent wars have shown that the use of infusion ratios of red blood cell and plasma concentrates should be between 1: 1 and 2: 1, with benefits in the mortality rate. The infusion of platelets is an important factor in coagulation and the replacement of fibrinogen critical, as soon as possible, when blood values are below $100 \mathrm{mg} / \mathrm{dL} .{ }^{32-34}$ The main objective of volume replacement is to increase plasma volume, correct coagulation disturbances and ensure tissue perfusion, with improvement of vascular rheology and acidosis correction. ${ }^{35-36}$

\section{Conclusion}

Due to the severity and high mortality of hypovolemic shock, the presence of an experienced and multidisciplinary team is essential. Capability of anticipating complications and act immediately can preserve lives. Hemodynamic monitoring is a fundamental part of the patient's treatment in shock, and knowing how to interpret data obtained and act on them is essential.

\section{Acknowledgements}

None.

\section{Conflict of interest}

There is no conflict of interest related to the completion of this study.

\section{References}

1. Sauaia A, Moore FA, Moore EE, et al. Epidemiology of trauma deaths: A Reassessment. Injury, Infection, and Critical Care. 1995;38(2):185-193.

2. Cecconi M, De Backer D, Antonelli M, et al. Consensus on circulatory shock and hemodynamic monitoring. Task force of the european society of intensive care medicine. Intensive Care Medicine. 2014;40(12):17951815.

3. Edul VSK, Ince C, Dubin A. What is microcirculatory shock? Current Opinion in Critical Care. 2015;21(3):245-252. 
4. Dutton RP. Current Concepts in hemorrhagic shock. Anesthesiology Clinics. 2007;25(1):23-34.

5. Nemet M, Tanczos K, Demeter G, et al. Central venous oxygen saturation and carbon dioxide gap as resuscitation targets in hemorrhagic shock. Acta Anaesthesiologica Scandinavica. 2014;58(5):611-619.

6. Tanczos K, Molnar Z. The oxygen supply-demand balance: a monitoring challenge. Best Practice and Research Clinical Anaesthesiology. 2013;27(2):201-207.

7. Tachon G, Harrois A, Tanaka S, et al. Microcirculatory alterations in traumatic hemorrhagic shock. Critical Care Medicine. 2014;42(6):14331441.

8. Maheshwari R, Subramanian RM. Severe acute pancreatitis and necrotizing pancreatitis. Critical Care Clinics. 2016;32(2):279-290.

9. Janisch NH, Gardner TB. Advances in management of acute pancreatitis Gastroenterologic clinics. 2016;45(1):1-8.

10. Cancio LC, Salinas J, Kramer GC. Protocolized resuscitation of burn patients. Critical Care Clinics. 2016;32(4):599-610.

11. Funk DJ, Jacobsohn E, Kumar A. The role of venous return in critical illness and shock-part I: Physiology. Critical Care Medicine. 2013;41(1):255-262.

12. Funk DJ, Jacobsohn E, Kumar A. The role of venous return in critical illness and shock: partII-Shock and mechanical ventilation. Critical Care Medicine. 2013;41(2):573-579.

13. Torres FIP, Torres LN, Pittman RN. Early physiologic responses to hemorrhagic hypotension. Translational Research. 2010;155(2):78-88.

14. Stahl W, Bracht H, Radermacher P, et al. Year in review 2009: Critica care - Shock. Critical Care. 2010;14(6):239-246.

15. Polanco PM, Pinsky MR. Practical issues of hemodynamic monitoring at the bedside. Surgical Clinics of North America. 2006;86(6):1431-1456.

16. McKinley BA, Sucher JF, Todd SR, et al. Central venous pressure versus pulmonary artery catheter directed shock resuscitation. Shock. 2009;32(5):463-470.

17. Suess EM, Pinsky MR. Hemodynamic monitoring for evaluation and treatment of shock: what is the current state of the art? Seminars in Respiratory and Critical Care Medicine. 2015;6(6):890-898.

18. McNeer RR, Varon AJ. Pitfalls od hemodynamic monitoring in patients with trauma. Anesthesiology Clinics. 2013;31(1):179-194.

19. Hata JS, Stotts C, Shelsky C, et al. Reduced mortality with noninvasive hemodynamic monitoring of shock. Journal of Critical Care. 2011;26(2):224.e1-8.

20. Antonelli M, Levy M, Andrews PJD, et al. Hemodynamic monitoring in shock and implications for management. International consensus conference, Paris, France, 27-28. Intensive Care Med. 2007;33(4):575590.
21. Pinsky MR. Hemodynamic evaluation and monitoring in the ICU. Chest. 2007;132(6):2020-2029

22. Jozwiak M, Monnet X, Teboul JL. Monitoring: from cardiac output monitoring to echocardiography. Current Opinion in Critical Care. 2015;21(5):395-401.

23. Molnar Z, Szabo Z, Nemeth M. Multimodal individualized concept of hemodynamic monitoring. Current Opinion of Anesthesiology. 2017;30(2):171-177.

24. Repesse X, Bodson L, Vieillard BA. Doppler echocardiography in shocked patients. Current Opinion in Critical Care. 2013;19(3):221-227.

25. McLean AS. Echocardiography in shock management. Critical Care. 2016;20:275-284.

26. Andritsos MJ, Park KW. Advantages and limitations of static parameters of fluid loading. International Anesthesiology Clinics. 2010;48:1-21.

27. Cavallaro F, Sandroni C, Antonelli M. Functional hemodynamic monitoring and dynamic indices of fluid responsiveness. Minerva Anestesiol. 2008;74(4):123-135.

28. Chang R, Holcomb JB. Optimal fluid therapy for traumatic hemorrhagic shock. Critical Care Clinics. 2017;33(1):15-36.

29. Moranville MP, Mieure KD, Santayana EM. Evaluation and managemen of shock states: hypovolemic, distributive, and cardiogenic shock. Journal of Pharmacy Practice. 2011;24(1):44-60.

30. Morrison CA, Carrick MM, Norman MA, et al. Hypotensive resuscitation strategy reduces transfusion requirements and severe postoperative coagulopathy in trauma patients with hemorrhagic shock: preliminary results of a randomized controlled trial. Journal of Trauma. 2011;70(3):652-663.

31. Cherkas D. Traumatic hemorrhagic shock: advances in fluid management Emergency Medicine Practice. 2011;13(11):1-20.

32. Spinella PC, Holcomb JB. Resuscitation and transfusion principles for traumatic hemorrhagic shock. Blood Reviews. 2009;23(6):231-240.

33. Nunez TC, Cotton BA. Transfusion therapy in hemorrhagic shock. Current Opinion in Critical Care. 2009;15(6):536-541.

34. Tien H, Nascimento B, Callum J, et al. An approach to transfusion and hemorrhage in trauma: current perspectives on restrictive transfusion strategies. 2007;50(3):202-209.

35. Cabrales $\mathrm{P}$, Tsai AG, Intaglietta $\mathrm{M}$. Is resuscitation from hemorrhagic shock limited by blood oxygen-carrying capacity or blood viscosity? Shock. 2007;27(4):380-389.

36. Zhao L, Wang B, You G, et al. Effects of different resuscitation fluids on the rheologic behavior of red blood cells, blood viscosity and plasma viscosity in experimental hemorrhagic shock. Resuscitation. 2009;80(2):253-258 